\title{
Bundling and Cooperation of Complementary Product
}

\author{
Lingzhi Shao \\ Institute of System Engineering, Southeast University, Nanjing, China \\ shaolingzhi2005@126.com
}

Keywords: Complementary products; Bundling strategy; Bundling effect; Cooperation

\begin{abstract}
We investigate product bundling strategy in a distribution channel with a common retailer and two complementary products suppliers. We get the thresholds of bundling effect for bundling strategy in different channel structures, and show that the cooperation of suppliers and retailer will improve the profitability of bundling strategy in some cases. The complementary suppliers cooperate to form an alliance only when the bundling effect is high enough, and the grand alliance could be stable when the allocation of bargaining power between channel members is appropriate. The bundle area will be expanded by the cooperation of retailer and by the cooperation of two suppliers when the degree of product complementarity is high enough.
\end{abstract}

\section{Introduction}

The concept of complementary products emerges when customers may have to buy more than one product at the same time to obtain the full utility of the products ([1]). Bundling the complementary products, the firms will provide their customers complete solutions for their thorny problems. [2] Found the combination of the degree of complementarities with which the bundling strategy is more benefit than components strategy. [3] Developed a profit-maximization model to provide a framework that can help firms optimizing bundling product categories and pricing strategies. [4] Investigated the strategic influence of product complementarity and advertising on the success of bundling products. When the most literatures focus on the bundling strategy of centralized firms, a few recent researches study bundling strategy in decentralized channel. For example, [5] analyzed the product bundling issues in a supply chain including a downstream retail and two manufacturers. He found that when the retailer sells several products from different manufacturers, he always prefer to separate sales, rather than bundling. [6] Examined how channel interactions influence product bundling decisions of channel members. They found that selling pure components by both manufacturer and retailer is the equilibrium except in a narrow region of the parameter space. [7] Got that bundling at the supply chain level is the most profitable, and the bundling area is the largest among all structures of channels.

In this paper, we will study the complementary products bundling strategy in the decentralized channels. However, there are several differences between our paper and the mentioned researches. Firstly, we focus on the product bundling strategy of complementary products and consider the bundling effect which reflect the addition of consumer's reservation price for bundles. Secondly, we will study the bundling strategy in the decentralized channels with different channel structures and investigate the effect of the cooperation of channel players on bundling strategy. Another difference of our study is that negotiation processes are introduced to calculate players' shares of cooperation pie. Although the Shapley Value is a classical way used to allocate the cooperation gains. But in practice, negotiation is a very widely observed mechanism used to define the allocation of the supply chain's profit among its players. There are many papers examining the supply chain decisions under the negotiation framework, for example [9-12]

The remainder of this paper is organized as follows: Section 2 introduces the problem and benchmark model about a completely non cooperative system; Section 3 and 4 analyze partial cooperative and fully cooperative channel, respectively; the summary is presented in section 5 . 


\section{Problem Description and Benchmark Model}

Problem Description. We consider a decentralized channel including two complementary suppliers and a common retailer who can choose retailing strategy: pure bundling or unbundling. In pure bundling setting, only the bundles of two complements 1 and 2 are offered to customers. In unbundling setting, the products are offered only separately. We assume that the retailer's size is larger than that of the two manufacturers, and the market is controlled by the monopolistic retailer. So in our benchmark model, two suppliers will interact with the retailer through Stackelberg game. However, the suppliers may cooperate to form an alliance. We call this situation the partial cooperation in Section 3. There also exist fully cooperative channel in Section 4 where the grand alliance including suppliers and retailer will be formed.

In the heterogeneity market whose size is 1 , the value of basic product 1 is represented by the customer's reservation price for that product $\left({ }^{r_{1}}\right)$ which indicates the maximum price he is willing to pay for it. From the retailer's perspective, the customers' reservation price for a product is a random variable whose distribution is uniform: $r_{1} \square U(0, a)$. We assume that the values of two complementary products in our model are perfectly positively correlated. So the reservation prices of complementary product 2 could be $r_{2}=(b / a) r_{1}, b \leq a$. The value of $(b / a)$ reflects the degree of product complementarity. The similar assumption has been made in other researches such as [13], [14]. In addition, it is reasonable to assume that: $c_{1}<a, c_{2}<b$. The bundling effect is denoted as $\theta$. Accounting to Venkatesh and Kamakura (2003)[2], when the consumer's reservation price for the bundle comprising one unit each of complementary products is denoted as $r_{b}$, we have $^{r_{b}}=(1+\theta)\left(r_{1}+r_{2}\right)$.

In unbundling setting, a customer buys any of the two products if the price of that product is not more than the customer's reservation price for it. In pure bundling setting, the customers whose reservation prices for bundle are more than bundle price will buy the bundle products. Hence, we can get the expected market segmentation for each product in unbundling setting and pure bundling setting, as follows: $D_{1}=\left(1-\frac{p_{1}}{a}\right), \quad D_{2}=\left(1-\frac{p_{2}}{b}\right) \quad D_{b}=\left(1-\frac{p_{b}}{(1+\theta)(a+b)}\right)$.

Benchmark Model----Bundling in a Decentralized Channel. We firstly introduce the benchmark model to study retailer's bundling strategy in a decentralized supply chain where each member makes their price decisions independently. In pure component setting, then we can get the equilibrium optimal solutions for pricing game in pure components setting:. In pure bundling setting, we can obtain the unique equilibrium sol $w_{1}^{n c}=\frac{a+3 c_{1}}{4}, w_{2}^{n c}=\frac{b+3 c_{2}}{4}, p_{1}^{n c}=\frac{3 a+c_{1}}{4}, p_{2}^{n c}=\frac{3 b+c_{2}}{4}$ unions for pricing game in pure bundling setting: $\quad w_{1}^{n b}=\frac{(a+b)(1+\theta)+5 c_{1}-c_{2}}{6}, \quad w_{2}^{n b}=\frac{(a+b)(1+\theta)+5 c_{2}-c_{1}}{6}, \quad p_{b}^{n b}=\frac{5(a+b)(1+\theta)+c_{1}+c_{2}}{6}$.we can get the proposition 1 as follows:

Proposition 1: if $\frac{c_{1}}{a}=\frac{c_{2}}{b}=\lambda$ and $0<\lambda<1$, the retailer will choose bundling strategy, when $\theta>\theta_{r}^{n b *}$, where $\theta_{r}^{n * *}=\frac{3(1-\lambda)^{2}+(1-\lambda) \sqrt{24 \lambda+9(1-\lambda)^{2}-4(1-\lambda)}}{4}$, otherwise he will sell the products separately.

We find that the retailer's bundling strategy will affect suppliers' performance. The supplier 1 who provides the main products may be damaged by retailer's bundling strategy, especially when the degree of product complementarity is low, i.e. $0<b / a<0.5$. But the complementary products supplier 2 will benefit from bundling strategy. 


\section{Bundling in a Partial-cooperative Channel}

In this section, we consider a partial-cooperative channel with the following stages: First, the suppliers decide whether to form an alliance selling a kit of products to retailer, and the retailer makes decision of bundling strategy. The two suppliers allocate their alliance profit using a cooperative bargaining procedure. Next, the suppliers' alliance trades with retailer in a Stackelberg game. Solving the game, we can get two suppliers' joint price and the retailing price in equilibrium as: $w_{1}+w_{2}=\frac{(1+\theta)(a+b)+3 c_{1}+3 c_{2}}{4}, \quad p_{b}=\frac{3(1+\theta)(a+b)+c_{1}+c_{2}}{4}$. Then there is $\Pi_{r}^{p b}>\Pi_{r}^{n u}$ for all $\theta>0$. So the retailer will adopt bundling strategy in partial-cooperative channel always.

But we need to check whether the partial-cooperative channel is stable. We assume that the shares of the "profit pie" that suppliers receive are calculated through some sort of negotiations among the parties. To model the negotiation process, we use the Generalized Nash Bargaining (GNB) Game: $\arg \max \left(x_{1}-d_{1}\right)^{\alpha}\left(x_{2}-d_{2}\right)^{\beta},\left(x_{1}, x_{2}\right) \geq d_{1}, d_{2} ; x_{1}+x_{2} \leq \Pi$. In our bargaining setting, with a pie $\prod_{s 1+s 2}^{p b}=\frac{\left[(1+\theta)(a+b)-c_{1}-c_{2}\right]^{2}}{16(1+\theta)(a+b)}, \prod_{s 1}^{p b}=\prod_{s 2}^{p b}=\frac{\left[(1+\theta)(a+b)-c_{1}-c_{2}\right]^{2}}{32(1+\theta)(a+b)}$ are profit allocation of suppliers with equal negotiation power $(\alpha=\beta=0.5$ ).

Finally, we reach the first step of the game which involves the two suppliers' cooperating decisions and retailer's bundling decision and get the following proposition 2.

$$
\begin{aligned}
& 0<-\frac{b}{a}<\frac{32 \lambda+16(1-\lambda)\left(3(1-\lambda)+\sqrt{9(1-\lambda)^{2}+24 \lambda}\right)-16 \lambda^{2}}{\left(3(1-\lambda)+\sqrt{9(1-\lambda)^{2}+24 \lambda}\right)^{2}} \\
& \theta>\frac{a(1-\lambda)^{2}+(1-\lambda) \sqrt{2 a(a+b) \lambda+a^{2}(1-\lambda)^{2}}-(a+b)(1-\lambda)}{(a+b)}
\end{aligned}
$$
the threshold for supplier's alliance and bundling strategy is

From the above proposition, the result shows that the suppliers' alliance may not be stable always, although all players and supply chain's bundle profit would be improved in partial cooperative setting comparing with no cooperative setting. Then we can further study the effect of cooperation on the bundling strategy and get the following Result 1 .

Result 1: The cooperation of suppliers may increase the profitability of retailer's bundling strategy, only when the degree of product complementarity is high enough.

\section{Bundling in a Fully-cooperative Channel}

With the participation of retailer, there are three settings: no cooperative channel as description in benchmark model; partial cooperative channel as studied in section 3; fully cooperative channel with grand alliance including retailer.

First of all, when all members cooperate to act as a centralized system, we can get the optimal solutions in component setting:

$$
p_{1}^{c u}=\frac{a+c_{1}}{2}, \quad p_{2}^{c u}=\frac{b+c_{2}}{2}, \text { and } p_{b}^{c b}=\frac{(a+b)(1+\theta)+c_{1}+c_{2}}{2} \text { in pure bundling setting. Bundling }
$$

strategy will be used when $\theta>0$, and the total profit pie is more than that in no and partial cooperative channel. Then we need to investigate the allocation of the profit pie between channel members, and check whether the grand alliance is stable.

We assume that the retailer and suppliers negotiate about the profit shares simultaneously. According to Multiplayer negotiation process, we will use Nash asymmetry negotiation model to get the allocation of profit pie which would be a family of solutions of the form: $\arg \max \left(x_{r}-d_{r}\right)^{\alpha}\left(x_{1}-d_{1}\right)^{\beta}\left(x_{2}-d_{2}\right)^{\gamma} ; \quad\left(x_{r}, x_{1}, x_{2}\right) \geq d_{r}, d_{1}, d_{2} ; x_{r}+x_{1}+x_{2} \leq \Pi_{s}^{c b}$. We still normalize the players' unequal disagreement point to zero, so the form of solutions can be rewrote as: 
$\arg \max \left(x_{r}\right)^{\alpha}\left(x_{1}\right)^{\beta}\left(x_{2}\right)^{\gamma}$. Keeping consistent with the other two channel structures, we let $\beta=\gamma=(1-\alpha) / 2$ and Then the allocation of profit pie could be got as: $\Pi_{r}^{c b}=\frac{\alpha\left[(1+\theta)(a+b)-c_{1}-c_{2}\right]^{2}}{4(1+\theta)(a+b)}$, $\Pi_{s 1}^{c b}=\Pi_{s 2}^{c b}=\frac{(1-\alpha)\left[(1+\theta)(a+b)-c_{1}-c_{2}\right]^{2}}{8(1+\theta)(a+b)}$

Proposition 3: If the bundling effect of two complementary products is quite obvious, then we have that: When $\frac{1}{2}<\alpha<\frac{3}{4}$, the grand alliance will be stable; when $\frac{1}{3}<\alpha<\frac{1}{2} \quad$ or $^{\frac{3}{4}<\alpha<1}$, the supplier's alliance will be stable instead of grand alliance. The products will always be sold as bundles by retailer or grand alliance.

Proposition 4: If the bundling effect of two complementary products is not significant, then we have that: When $\frac{(1+\theta)(1-\lambda)^{2}}{2(1+\theta-\lambda)^{2}}<\alpha<1-\frac{a(1+\theta)(1-\lambda)^{2}}{2(a+b)(1+\theta-\lambda)^{2}}$, the grand alliance including retailer will be stable and bundling strategy would be used by grand alliance; when $\frac{1}{3}<\alpha<\frac{(1+\theta)(1-\lambda)^{2}}{2(1+\theta-\lambda)^{2}}$ or $1-\frac{a(1+\theta)(1-\lambda)^{2}}{2(a+b)(1+\theta-\lambda)^{2}}<\alpha<1$, there is no alliance in channel, and products are sold by retailer as components.

Overall, the grand alliance could be stable, when the allocation of bargaining power between channel members is appropriate. The performance of supply chain would be the best in fully cooperation channel among all the three channel structures. Comparing the four propositions, we can get the following Result 2.

Result 2: The cooperation of retailer enhances the profitability of bundling strategy by expanding the bundle area.

\section{Conclusion}

In this paper, we try to analyze the synthesized effect of bundling effect and players' cooperation on complementary product bundling strategy. In a completely decentralized channel, the strong retailer will choose bundling strategy to improve his profit when the bundling effect is higher than a threshold. The supplier 2 who supply complementary products will also benefit from the retailer's bundling strategy. But the supplier 1 may be harmed in pure bundling setting, especially when the degree of product complementarity is quite low. The bundling area for retailer will be expended through suppliers' cooperation, when and only when the degree of product complementarity is high enough. We also show that the cooperation of retailer enhances the profitability of bundling strategy.

Our research could be extended from different perspectives. First, in this research we only considered pure bundling behavior, and it should be possible to extend our research to mixed bundling scenario in a supply chain. Second, to keep the model tractable, we made the assumptions that the reservations of two complementary products are completely positively correlated. So the analysis should also be extended to the situations in which the full spectrum of correlations will be considered. Third, it is possible and meaningful to extend our research to the more complex distribution channel which includes more than two suppliers.

\section{References}

[1] Yue X, Mukhopadhyay S K, Zhu X., A Bertrand model of pricing of complementary goods under information asymmetry, Journal of Business Research, 59(10): 1182-1192, 2006.

[2] Venkatesh R., Kamakura W., Optimal bundling and pricing under monopoly: Contrasting complements and substitutes from independently valued products, Journal of Business, 76(2): 211-231, 2003. 
[3] Yan R, Bandyopadhyay S., The profit benefits of bundle pricing of complementary products, Journal of Retailing and Consumer Services, 18(4): 355-361, 2011.

[4] Chris Myers, Ruiliang Yan, John Wang, Sanjoy Ghose., Bundling products to success: The influence of complementarity and advertising, Journal of Retailing and Consumer Services, 21: 48-53, 2013.

[5] Bhargava, Hemant K., Retailer-Driven Product Bundling in a Distribution Channel, Marketing Science, 31 (6): 1014-1021, 2012.

[6] Girju M., Prasad A., Ratchford B T., Pure Components versus Pure Bundling in a Marketing Channel, Journal of Retailing, 89(4): 423-437, 2013.

[7] Chakravarty A., Mild A., Taudes A., Bundling decisions in supply chains, European Journal of Operational Research, 231(1): 617-630, 2013.

[8] Feng Qi, and Lauren Xiaoyuan Lu, Supply chain contracting under competition: Bilateral bargaining vs. stackelberg, Production and Operations Management, 22 (3): 661-675, 2013.

[9] Lin R.Y., Kong L.Z., The impact of channel power of symmetric competing channels on the profit segmentation, In: Proceeding of National Conference of Chinese National Academy, December 21, 2002.

[10] Sheu Jiuh-biing, Bargaining framework for competitive green supply chains under governmental financial intervention, Transportation Research Part E, 47(5):573-592, 2011.

[11] Sheu Jiuh-biing and Xiao-qin GAO, Alliance or no alliance: Bargaining power in competing reverse supply chains, European Journal of Operational Research, 233 (2): 313-325, 2014.

[12] McCardle K. F., Kumar R., \& Tanga C. S., Bundling retail products: Models and analysis. European Journal of Operational Research, 177(1): 1197-1217, 2007.

[13] Sheikhzadeh Mehdi, Elahi Ehsan, and Product bundling: Impacts of product heterogeneity and risk considerations, International Journal of Production Economics, 144(1): 209-222, 2013.

[14] Timothy Derdenger, Vineet Kumar, the Dynamic Effects of Bundling as a Product Strategy, Marketing Science, 32(6):827-859. 2013. 